Revue des patrimoines

$17 \mid 2011$

Les patrimoines de l'enseignement supérieur

\title{
Le patrimoine écrit des universités : les débuts d'une reconnaissance
}

\section{Charlotte Maday et Stéphanie Méchine}

\section{(2) OpenEdition}

Journals

Édition électronique

URL : http://journals.openedition.org/insitu/5504

DOI : 10.4000/insitu.5504

ISSN : 1630-7305

Éditeur

Ministère de la culture

Référence électronique

Charlotte Maday et Stéphanie Méchine, «Le patrimoine écrit des universités : les débuts d'une reconnaissance », In Situ [En ligne], 17 | 2011, mis en ligne le 28 mars 2012, consulté le 19 avril 2019. URL : http://journals.openedition.org/insitu/5504; DOI : 10.4000/insitu.5504

Ce document a été généré automatiquement le 19 avril 2019

\section{(c) $($ i) $(9)$}

In Situ Revues des patrimoines est mis à disposition selon les termes de la licence Creative Commons Attribution - Pas d'Utilisation Commerciale - Pas de Modification 4.0 International. 


\title{
Le patrimoine écrit des universités : les débuts d'une reconnaissance
}

\author{
Charlotte Maday et Stéphanie Méchine
}

1 Depuis peu, les archives des universités françaises suscitent l'intérêt de la communauté scientifique, notamment des historiens, des géographes et des sociologues ${ }^{1}$. Malgré de nombreuses menaces ${ }^{2}$ et la perte irrémédiable de certains fonds, ce patrimoine écrit a cependant été sauvegardé dans sa très grande majorité grâce à des actions menées par des professionnels de la conservation tant dans les centres d'archives que dans les bibliothèques d'études et de recherche à Paris ou, dans les grandes villes de province, des bibliothèques municipales et universitaires qui jouent souvent ce même rôle ${ }^{3}$.

2 Les producteurs de ces archives, les universités elles-mêmes, célèbrent aujourd'hui pour la plupart leur $40^{\mathrm{e}}$ anniversaire et sont désormais beaucoup plus sensibles à la question de la préservation de leur patrimoine tant pour des besoins administratifs et juridiques que pour des raisons historiques. En effet, celui-ci consiste en une multitude de dossiers, registres, plans ou fichiers, sur des aspects aussi variés que le fonctionnement des institutions universitaires (à travers notamment les procès-verbaux de leurs conseils ou ceux de leurs laboratoires), la scolarité, la gestion du personnel, la comptabilité ou encore les opérations de construction et de maintenance liées aux bâtiments.

Ce premier constat nous conduira à développer les aspects historiques liés à la constitution du fonds de l'Université de Paris par le rectorat de Paris, puis à présenter l'action majeure du réseau professionnel d'archivistes Aurore ${ }^{4}$ dans le domaine de la collecte et de la conservation de ces archives universitaires et enfin, plus particulièrement, les travaux de l'un de ses groupes de travail sur les archives universitaires en Île-de-France. 


\section{Constat et constitution d'un patrimoine archivistique universitaire en Île-de-France}

4 Les aspects historiques liés à la constitution et à la conservation du patrimoine archivistique des universités de l'Ancien Régime, puis celles refondées ou créées entre le I er Empire et 1970, mériteraient une analyse fine que l'on résumera à grands traits pour la partie des collections conservées dans des centres d'archives. Si la lettre circulaire parue en 1932 et adressée aux « recteurs d'académie [...] a prescrit sous certaines conditions le versement aux Archives départementales des archives existant auprès des organismes universitaires [des départements] jusqu'à l'année $1890 »^{5}$, il faut attendre la diffusion des résultats de deux enquêtes réalisées, l'une en 1966 et l'autre en 1981, pour disposer d'un état des collections des archives publiques des universités dans les services d'archives définitives. Leur objectif était de mesurer, pour la première, l'impact du décret du 10 juillet $1936^{6}$ sur l'obligation de versement par tous les ministères, administrations, services et établissements de l'État et sur l'exercice du contrôle des éliminations d'archives et, pour la seconde, l'application de la circulaire ministérielle de 1970 sur les archives de l'enseignement?

\section{Diffusion d'enquêtes nationales}

La première enquête, qui résultait d'une collaboration entre l'Association des archivistes français et la Direction des Archives de France ${ }^{8}$, soulignait, pour les universités, la rareté des versements d'archives conservées alors dans les services d'archives départementales.

6 La seconde enquête, menée quinze ans plus tard par la mission des Archives nationales auprès du ministère de l'Éducation nationale, présentait un bilan de dix ans de versements d'archives des administrations et des établissements, résumé ici pour la partie relative à l'enseignement supérieur'. Cette enquête, commentée à l'occasion d'une journée d'étude à Aix-en-Provence ${ }^{10}$ et dans les actes d'une autre journée tenue à Nancy ${ }^{11}$, faisait suite aux conclusions d'une commission tripartite dirigée, en 1969, par Pierre Renouvin ${ }^{12}$. Composée de quatre conservateurs, deux historiens, Ernest Labrousse et Paul Gerbod, et deux inspecteurs généraux des archives, son objectif était de poser les bases d'une réflexion sur le sort des archives contemporaines de l'enseignement ${ }^{13}$. L'enquête de 1981 signalait, pour les universités, le versement de quarante-quatre établissements dans vingt départements, soit en moyenne 2,2 établissements par département, émanant des facultés des universités (des lettres, droit et sciences économiques, médecine, sciences et pharmacie), et concluait, pour ces établissements, au versement global de leurs archives anciennes en application de la circulaire de 1970. L'enquête apportait une précision supplémentaire en indiquant que, sur l'ensemble des quarante-quatre établissements, sept seulement avaient versé des documents couvrant l'ensemble de la période considérée, qui s'étendait de l'Empire à la fin des années 1970.

7 Les catégories de documents versés par ces universités aux Archives départementales avaient été regroupées sous quatre grandes rubriques reprises ici: administration générale, scolarité, personnel et intendance (respectivement représentées dans 25, 35, 15 et 5 établissements). Concernant l'administration générale, la plus forte représentation revenait aux procès-verbaux des conseils et assemblées de professeurs (14 cas sur 25) qu'on peut également retrouver dans les fonds des rectorats, et aux budgets et pièces 
financières ou comptables (13 cas sur 25). On trouvait ensuite les rapports des doyens au ministre sur la situation de l'établissement, les procès-verbaux et discours des séances de rentrée, la correspondance reçue et envoyée et les collections d'arrêtés rectoraux. La rubrique scolarité recensait les registres d'inscription et d'immatriculation, les dossiers d'étudiants ainsi que les pièces portant sur l'organisation et la sanction des examens (26 cas sur 35), ainsi que les dossiers de bourses. Pour le personnel, avaient été retenus pour l'essentiel les dossiers individuels de personnel, les dossiers des élèves-professeurs à l'institut préparatoire à l'enseignement secondaire (IPES) et les pièces justificatives de traitements des fonctionnaires. La dernière catégorie intitulée intendance englobait les dossiers de constructions universitaires et les inventaires du mobilier et des collections.

8 Était notée, en revanche, l'absence quasi-totale de dossiers, pourtant répertoriés par la circulaire de 1970, "touchant à l'organisation générale de l'enseignement et de la recherche, tels que procès-verbaux et dossiers d'instituts, les dossiers scientifiques de laboratoires, les papiers privés de professeurs, etc. $»^{14}$

\section{Les résultats de la collecte des archives des facultés de l'Université de Paris et des universités héritières}

Pour les archives universitaires, le cas du rectorat de Paris mérite un examen plus approfondi. En premier lieu, l'ensemble des "fonds facultaires", de la création de l'Université de Paris à son éclatement à la suite de l'application de la loi Faure, a été versé aux Archives nationales ${ }^{15}$. Ce résultat s'explique par le fait que le rectorat de Paris, par dérogation à la règle commune, a bénéficié d'une mission des Archives nationales implantée dans le quadrilatère de la Sorbonne avec mission, d'une part, de classer et verser les dossiers entassés par les services rectoraux dans le dépôt conçu par Henri-Paul Nénot et, de l'autre, de mener le même type d'opération dans les facultés occupant les locaux de la Sorbonne et à proximité. (fig. $\mathbf{n}^{\circ} \mathbf{1}$ ) 


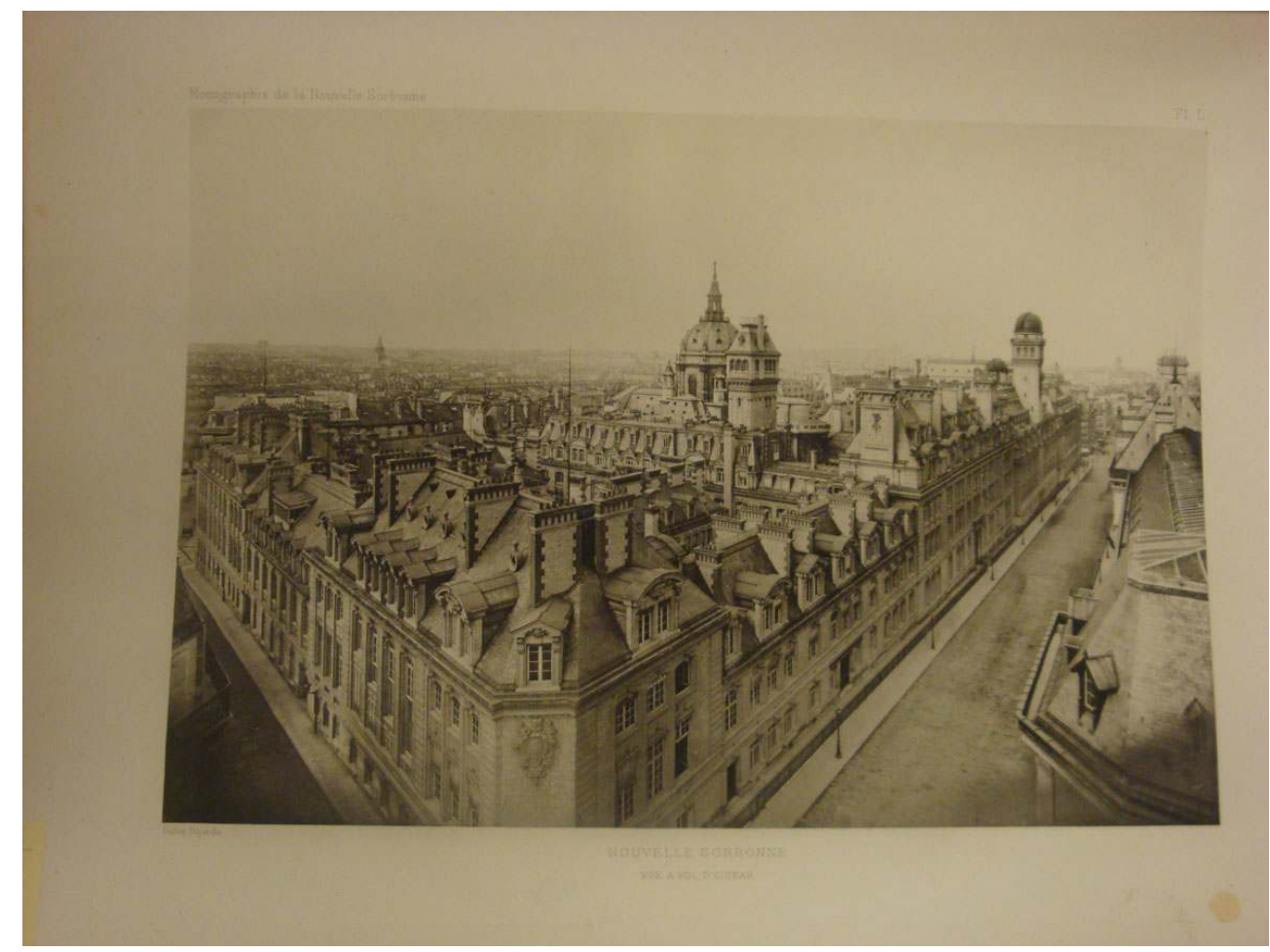

Extrait d'un ouvrage d'H.-P. Nénot, Monographie de la nouvelle Sorbonne, Paris, Imprimerie nationale, 1902.

10 L'ensemble des dossiers a été versé aux Archives nationales à Paris, d'abord dans la soussérie $\mathrm{AJ}^{16}$, créée spécialement pour les accueillir à partir de 1935, puis, pour la seconde moitié du $\mathrm{XX}^{\mathrm{e}}$ siècle, à Fontainebleau où ils sont conservés pour partie sous des cotes à dix chiffres ${ }^{16}$.

11 S'il est vrai que le contenu des archives de la faculté des lettres se résume, pour l'essentiel, aux dossiers concernant l'administration, le personnel et la scolarité, celles émanant de la faculté des sciences et de pharmacie sont cependant plus riches. En effet, on y trouve des dossiers relatifs aux laboratoires, instituts et fondations de 1911 à 1946, des rapports sur les candidats aux chaires de 1884 à 1927 ou encore des affiches de coursprogrammes de 1853 à 1928.

En outre, dans le fonds de la faculté de pharmacie sont conservés les dossiers de réforme des études et des structures d'enseignement et de recherche (rapports, projets, textes législatifs et réglementaires) de 1935 à 1970 et de contrôle de l'exercice de la pharmacie au travers notamment de procès-verbaux de réunions et d'enquêtes rédigés entre 1911 et 1966.

13 Le fonds de la faculté de médecine de Paris, quant à lui, est exceptionnel, tant en raison de la longévité et de l'exhaustivité des séries d'archives relatives aux grandes catégories citées ci-dessus, qu'en raison de la conservation de certains dossiers tels que les rapports, décrets et arrêtés relatifs aux écoles de santé de Paris, Strasbourg et Montpellier de l'an III à l'an VIII, ou encore ceux concernant les expositions, les congrès, les relations internationales, les cérémonies, les sociétés savantes, les associations, ainsi que les questions générales d'enseignement et travaux des professeurs de l'an III à 1938. Enfin, de 
même que pour le fonds de la faculté des sciences de Paris, on y trouve également des archives relatives aux laboratoires, instituts, archives, musées, bibliothèques et collections, de l'an III à 1949.

14 Ce résultat n'a rien d'étonnant, dans la mesure où l'on sait que du personnel a été diligenté pour assurer une gestion des archives dans deux de ces trois facultés (médecine et pharmacie) et que, pour la première, un archiviste paléographe a conservé, trié et classé les dossiers qui lui ont été confiés au XIX ${ }^{\mathrm{e}}$ siècle, assurant par-là la préservation du fonds de la partie la plus ancienne jusqu'à son classement définitif, un siècle plus tard ${ }^{17}$. (fig. $n^{\circ} 2$ )

Figure 2

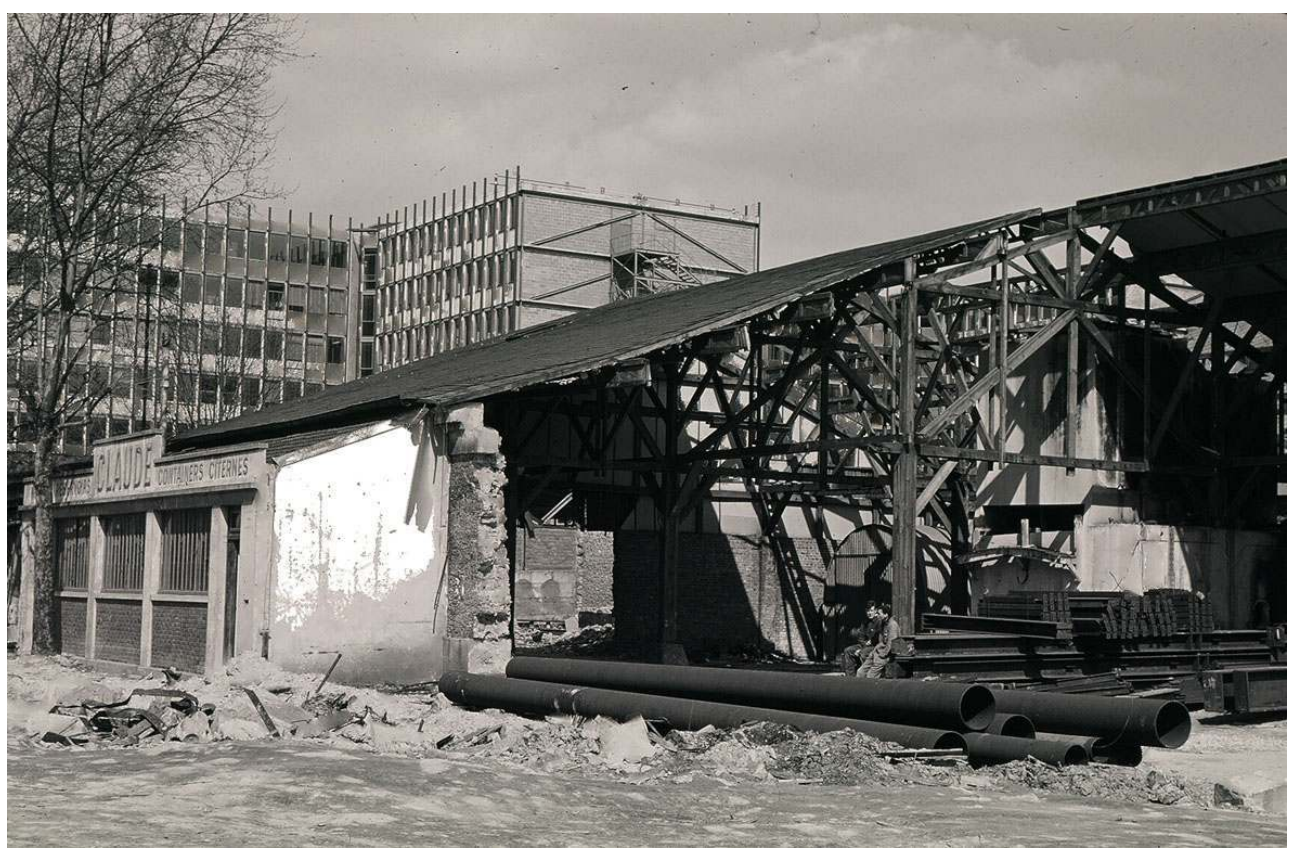

Archives de Paris Diderot, série Ph (ph 6-5) «Faculté des sciences : destruction de la Halle aux Vins, construction de Jussieu » par M. Pascal (1964-1969).

(c) Université Paris Diderot.

De même, pour la période contemporaine, sont consultables à Fontainebleau, à titre d'exemple, dans les archives de la présidence de l'université Paris- $\mathrm{V}$, les dossiers relatifs à la création et à l'organisation de cet établissement ou encore celles de la faculté Neckerenfants malades ${ }^{18}$ [qui renseignent sur la construction de ce bâtiment dont l'architecte est André Wogenski, disciple de Le Corbusier]. Cependant, ces archives sont pour l'essentiel constituées de documents à caractère administratif qui ne donnent pas de renseignements précis sur le contenu des enseignements dispensés, de la recherche et de leur réception par les étudiants. Il faut donc avoir recours à d'autres fonds d'archives publiques tels que ceux des enseignants-chercheurs et des laboratoires. Par exemple, signalons, pour une autre discipline, les dossiers de Marguerite Boulet-Sautel et de Gérard Sautel, professeurs à la faculté de droit puis à l'université Paris-II, qui se révèlent riches en informations sur la création des UFR de droit sur le territoire francilien au moment de la mise en place de la loi d'orientation sur l'enseignement supérieur ${ }^{19}$ et, pour les disciplines telles que les mathématiques, les dossiers des laboratoires de la faculté des 
sciences puis des universités Paris-VI et Paris-VII ${ }^{20}$, sauvegardés lors de la campagne de désamiantage.

\section{Un complément indispensable : les archives de tutelle du rectorat de Paris}

16 À ce patrimoine archivistique, s'ajoutent en premier lieu les archives de tutelle du ministère de l'Éducation nationale, également conservées dans les centres de Paris et de Fontainebleau des Archives nationales, ainsi que les dossiers de tutelle du rectorat, d'une exceptionnelle qualité à Paris pour la période s'étendant de 1870 à 1970 environ ${ }^{21}$. Dans la sous-série $\mathrm{AJ}^{16}$ signalons, pour les services académiques, l'existence des dossiers des conseils de facultés et du conseil académique de 1883 à 1939, comportant les procèsverbaux de séances et documents préparatoires. On y trouve également les archives portant sur la création et le fonctionnement des instituts français à l'étranger ou sur les relations internationales de l'université, telles que les dossiers relatifs à la participation de délégations françaises à des congrès internationaux, à l'invitation de professeurs étrangers. Enfin sont également conservées les archives relatives au baccalauréat entre 1820 et 1946.

Sur le site de Fontainebleau est conservé le fonds d'archives administratives relatif à l'enseignement supérieur de 1870 à 1970 environ. Il regroupe un grand nombre de dossiers sur les facultés de l'université de Paris et ses instituts comme ceux, pour les sciences humaines et sociales, de l'ethnologie et, pour partie, de la psychologie, de la géographie et de l'histoire de l'art, ou encore, pour les sciences "dures ", des instituts de chimie ou du radium ${ }^{22}$. On y trouve également les dossiers de construction d'établissements universitaires tels que ceux de la Sorbonne et des trois facultés environnantes, médecine, droit et pharmacie.

Pour la période postérieure à 1970, à quelques exceptions près, les archives du rectorat de Paris sont désormais versées aux Archives de Paris ${ }^{23}$.

Nous achèverons cette présentation par l'énumération d'autres fonds en précisant toutefois qu'il ne s'agit plus d'archives de tutelle mais de compléments indispensables à l'histoire des étudiants. Le service des archives mettra bientôt à disposition l'instrument de recherche établi à la suite du classement de nombreux dossiers émanant des services du CROUS parisien, riches en informations depuis sa création en 1936 jusqu'aux années 1970. L'étude de ce fonds pourra être complétée par la consultation des archives du bureau universitaire de statistiques conservées aux Archives nationales en $63 \mathrm{AJ}$, du sport scolaire et universitaire conservées en $69 \mathrm{AJ}$, regroupant les dossiers du sport scolaire et universitaire (OSSU) créé en 1934 et de l'association du sport scolaire et universitaire (ASSU) qui lui a succédé en 1963. On ajoutera à cette liste les archives du Centre national des œuvres universitaires et scolaires (CNOUS), établissement public administratif dont on trouve plusieurs versements à Fontainebleau et celles des associations ${ }^{24}$ pour lesquelles nous renvoyons les lecteurs vers les institutions qui les conservent.

20 Les résultats de cette collecte, portés à la connaissance du public à différentes occasions [grâce au dynamisme d'une mission des Archives nationales transformée en service d'archives autonome], continuent de s'enrichir [...]. Le rectorat poursuit son action de sensibilisation à ce patrimoine écrit, soit de manière directe auprès de certaines universités, soit en préconisant la création de services d'archives dans les établissements 
qui n'en sont pas encore dotés, et ce dans le prolongement de l'application de la circulaire du 2 novembre $2001^{25}$.

\section{Déploiement et harmonisation de la collecte au sein des établissements universitaires franciliens}

\section{Archives universitaires en péril ? : premier état des lieux}

Depuis leur création en 1970 par la loi Faure ${ }^{26}$, les universités franciliennes, succédant aux facultés de l'Université de Paris, n'ont pas eu l'opportunité ou n'ont pas eu l'occasion de mesurer les enjeux d'une politique documentaire efficace telle qu'elle est pratiquée depuis plusieurs décennies dans certaines universités européennes et américaines, alors même que les circulaires de 1970 et de $1975^{27}$ rappelaient l'importance et le besoin de classer et de conserver ces documents.

Ces raisons ont eu pour effet une absence de sensibilisation des personnels des universités aux obligations réglementaires et à la préservation de l'histoire écrite de leur établissement et ont conduit les services administratifs, les laboratoires et les enseignants-chercheurs à gérer seuls leurs documents.

Lorsque l'archiviste nouvellement en poste en université procède à des audits et des récolements sur les fonds d'archives dont il a la charge dans les espaces de stockage, il constate que dans un certain nombre de cas, les archives ont été malmenées, oubliées, quand bien même certaines d'entre elles renfermeraient des éléments d'information indispensables à la justification des actions menées par l'établissement, et à l'histoire de la recherche, de l'administration, des personnels, des étudiants, des bâtiments et de leur entretien. Ces situations, pour certaines catastrophiques, ont cependant pour avantage de rendre compte du rapport que peut entretenir chaque fonctionnaire, chaque personnel, avec ses documents de travail.

\section{Implantation et action des services d'archives dans les universités : la création d'un réseau}

Depuis quelques années, les universités franciliennes sont soumises à des défis majeurs ayant des implications en matière de production, de gestion et de conservation d'archives, qui sont à l'origine de la création de postes d'archivistes.

La première raison qui prévaut à leur existence est très certainement due aux problèmes de gestion de l'espace, particulièrement prégnants dans l'environnement parisien. En effet, les principaux locaux des universités issues de la loi Faure sont ceux qui abritaient les anciennes facultés ou leurs annexes (Orsay, Nanterre et, à Paris, Jussieu, Censier, Tolbiac, Dauphine ainsi que Vincennes qui a été déplacée à Saint-Denis à la fin des années 1970). (fig. $\mathbf{n}^{\circ} \mathbf{3}$ ) 
Figure 3

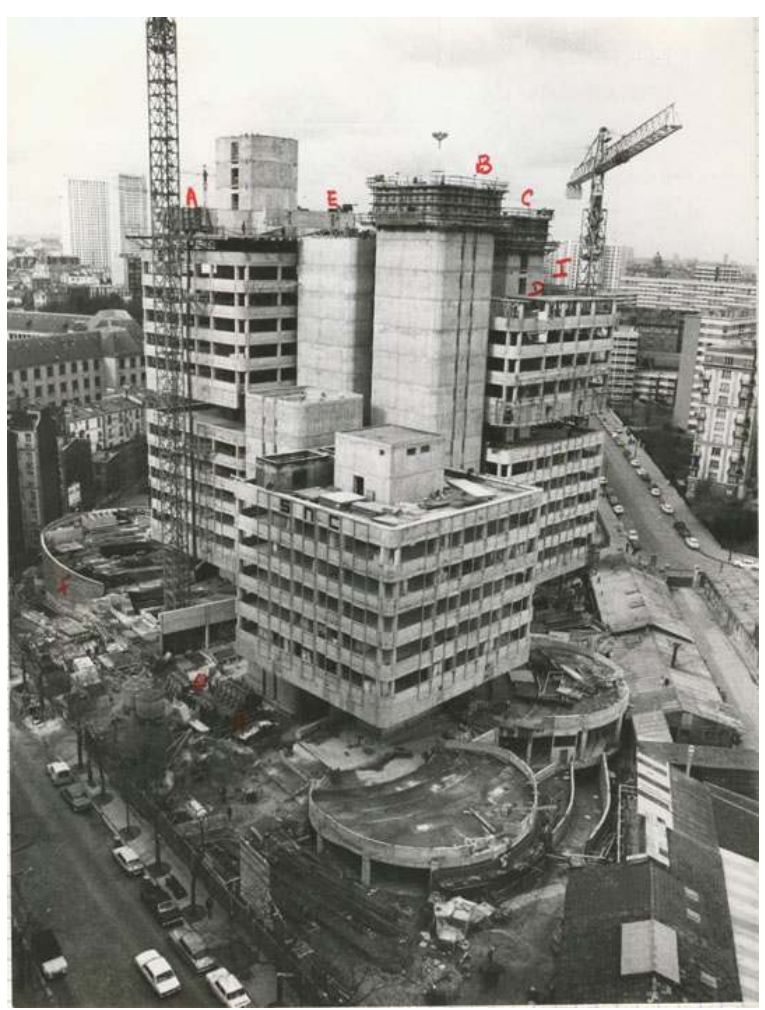

Vue d'ensemble de la construction de Tolbiac, extrait du fonds du service constructeur des académies de la région Île-de-France (SCARIF), 24 février 1973, cote provisoire SCARIF/Tolbiac/carton 6.

(c) SCARIF.

Depuis lors, ces universités ont fait construire à leur tour de nouveaux bâtiments ${ }^{28}$ ou de nouvelles « annexes » ou ont loué des bâtiments dont la liste ${ }^{29}$ est si longue que nous ne sommes pas en mesure de la dresser ici. Celles qui sont implantées depuis l'origine dans des locaux de la fin du XIXe siècle (Paris-I, Paris-II, Paris-IV, Paris-V) sont dotées d'espaces de stockage souvent mal adaptés à une bonne conservation des archives.

La deuxième est d'ordre archivistique : la circulaire de $2005^{30}$ a rappelé aux responsables des universités, présidents comme secrétaires généraux, l'importance fondamentale d'une bonne gestion des archives et a approfondi le cadre réglementaire en matière de tri et de classement des documents.

La troisième est liée au contexte actuel de l'enseignement supérieur et de la recherche, issu de la nouvelle organisation administrative qui fait suite à l'application de la loi relative aux libertés et responsabilités des universités (LRU) ${ }^{31}$, votée en 2007 . Cette loi prévoit que, dès le $1^{\mathrm{er}}$ janvier 2013, les établissements universitaires dans leur ensemble deviennent autonomes dans les domaines de la gestion des ressources humaines, de la gestion financière, et pour ceux qui en font la demande, de la gestion de leur patrimoine immobilier.

29 La loi LRU a modifié profondément le mode de gestion des établissements et leur organisation: certaines universités, pressentant ce bouleversement avaient, dès 2005, projeté de modifier leur structure administrative pour anticiper au mieux les conséquences de la transition entre un système de gestion administratif et celui qu'elles devraient adopter dès 2013. Quelques responsables avaient mesuré la nécessité de 
s'associer un gestionnaire de l'information, organisateur de la collecte d'archives et responsable de l'harmonisation de la production documentaire.

Les universités franciliennes sont actuellement presque toutes dotées de services d'archives, et le processus de recrutement de spécialistes s'est accéléré depuis 2010 tant au niveau local qu'au niveau national. Pour revenir à l'île-de-France, l'université ParisVII est la première à s'être dotée d'un service d'archives, en 2002 et ce pour des raisons juridiques $^{32}$. L'université Paris-IX est la suivante à recruter un archiviste en 2005, puis c'est au tour de Paris-X d'en faire autant en 2007, suivie de Paris-VI et Paris-I en 2008, puis de Paris-VIII en 2010 et enfin de Paris-V et de Paris-XII en 2011. (fig. $\mathbf{n}^{\circ} \mathbf{4}$ )

Figure 4

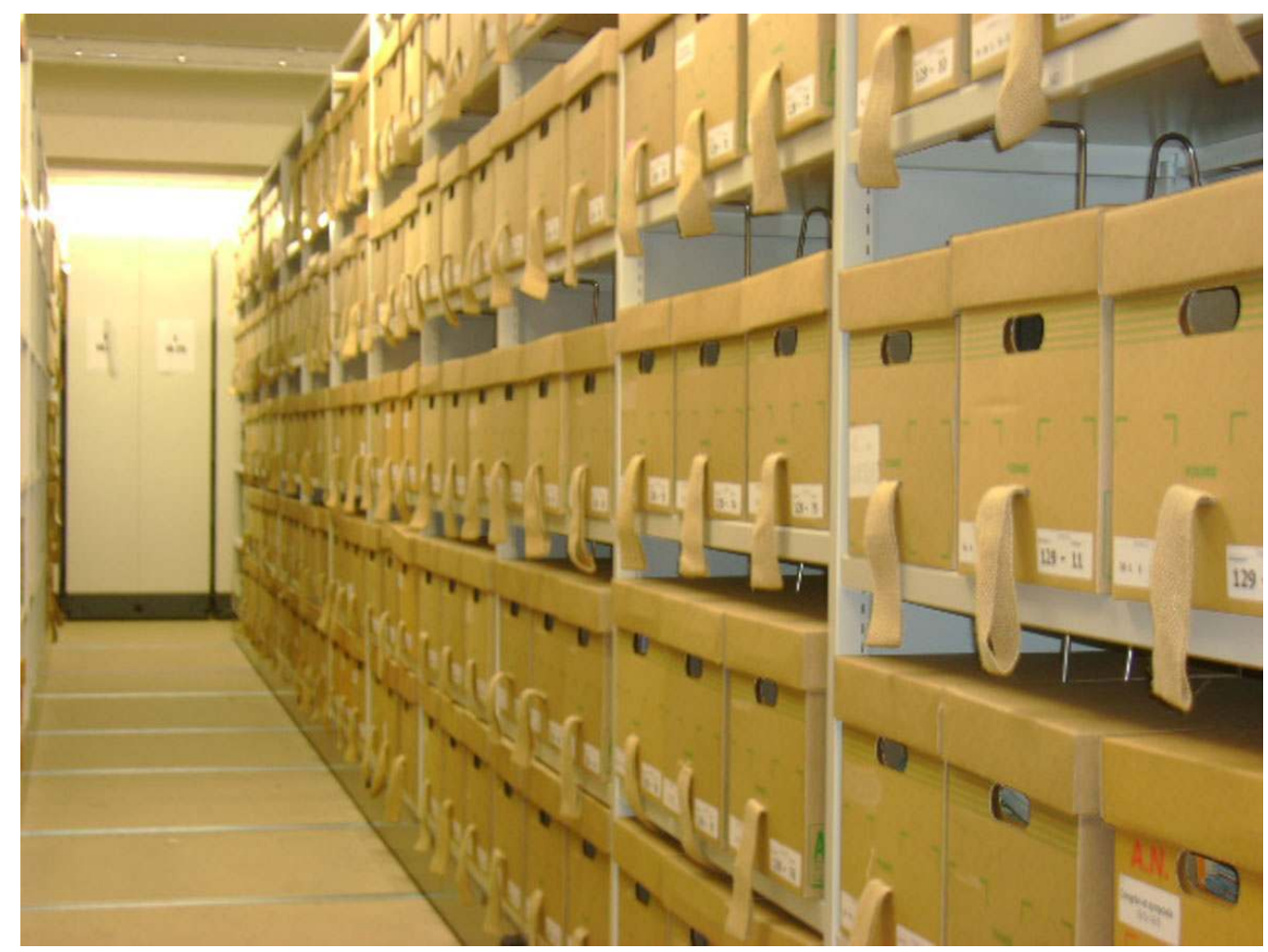

Magasins d'archives, université Paris Diderot-Paris 7. Phot. Olivier Azzola, 2008.

(c) Université Paris Diderot.

31 Au-delà des opérations relevant de la compétence du professionnel des archives, ainsi que des actions de tri et de classement des archives des services administratifs ${ }^{33}$, ou de celles des instances ${ }^{34}$, les archivistes des universités sont parvenus à faire reconnaître d'autres actions, plus délicates à mener dans le contexte actuel des établissements, mais tout aussi légitimes. Parmi celles-ci, on peut citer quelques opérations de collecte des archives scientifiques et la mise au point de procédures incluant la sensibilisation et la formation aux bonnes pratiques de classement d'archives auprès des personnels scientifiques, la réalisation ou la participation à des projets de valorisation du patrimoine comme la collecte de témoignages oraux, la préparation d'expositions, de communications à des journées d'étude ou encore la participation à des ouvrages sur des sujets ayant trait à la mémoire et à l'histoire de certains établissements universitaires.

32 L'intervention de l'archiviste à tous les niveaux (administratif, pédagogique, et scientifique), fait de lui un acteur formé pour identifier l'ensemble des rouages de son 
administration et un pourvoyeur d'aide indispensable aux publics et notamment aux chercheurs qui travaillent à l'écriture de l'histoire des universités.

En 2007, le réseau Aurore, rassemblant les archivistes des établissements d'enseignement supérieur et des rectorats (archives de tutelle de l'enseignement supérieur) sur le territoire national, est créé pour répondre au besoin de partage de pratiques et ainsi mener une réflexion de fond en termes de stratégie de collecte et d'aide et de soutien aux archivistes nouvellement recrutés et exerçant dans le contexte professionnel de l'enseignement supérieur et la recherche.

Lancé en 2009, le groupe de travail sur les archives des universités de Paris est la première application locale, sur un espace géographique donné, de la réflexion menée au niveau national, et résulte de l'histoire particulière qui unit le rectorat de Paris aux universités de son ressort. Ce groupe, qui s'est élargi en 2010 à l'île-de-France, rassemble aujourd'hui les archivistes des universités de Paris I, de Paris-V à Paris X, de Paris XII et des interlocuteurs de Paris II et Paris III autour de ceux du rectorat de Paris ${ }^{35}$.

D'abord centrée sur la définition d'actions en faveur de la gestion des archives en université (archives de la scolarité, archives et dossiers de personnels pour l'essentiel), sur les problématiques d'archivage électronique et la gestion électronique de documents (GED), le groupe de travail a, par la suite, cherché à élaborer une stratégie de valorisation des sources archivistiques conservées en établissement, dans la perspective notamment de préparer des manifestations autour des célébrations du $40^{\mathrm{e}}$ anniversaire des universités franciliennes.

À partir de premières réflexions, enrichies bien vite par des enseignants-chercheurs, ou parallèlement à celles-ci, des actions ont été menées soit au niveau local de chaque établissement, soit en association, en vue d'améliorer la visibilité des structures d'archives au sein des universités, pour parvenir à renforcer le soutien hiérarchique aux actions de valorisation du patrimoine, pour animer le lien entre enseignants chercheurs et archivistes en sensibilisant sur le métier et, enfin, pour contribuer à soutenir la recherche en histoire de l'enseignement et des disciplines, avec la mise à disposition de sources. À travers l'organisation de journées d'études en 2011 et 2012 et d'un colloque en 2013 destinés à jalonner les comptes rendus de travaux de classement et de mise a disposition de documents d'archives, le but est aussi d'obtenir des moyens pour les travaux de classement et de valorisation : création et mise en ligne de bases de données, parallèlement au projet de guide des sources Aurore, développement des travaux de recherche pouvant alimenter ce guide.

\section{Conclusion}

37 Les collections d'archives des facultés de l'Université de Paris et une partie de celles produites par les universités entre 1970 et 2000 sont venues enrichir les Archives nationales et, depuis peu, les Archives de Paris, grâce aux professionnels de la conservation du rectorat de Paris, et ce durant près de 75 ans. Les approches et actions successives menées auprès des établissements parisiens n'ont cependant par permis de mettre en place une politique de collecte et de gestion des archives courantes et intermédiaires. Ce n'est qu'à partir du moment où des professionnels des archives ont été recrutés par les établissements que la collecte et la préservation de ces documents témoignant non seulement du fonctionnement d'institutions indispensables à la société, 
mais également du dynamisme de la recherche en France, a pu s'effectuer, permettant ainsi de mettre en place des procédures, de poursuivre la mise à disposition de nouvelles sources et de faire connaître la richesse de ce patrimoine écrit.

Le visage de la recherche a aujourd'hui profondément changé : le regroupement des universités et des établissements de recherche dans des pôles de recherche et d'enseignement supérieur ainsi que l'émergence de fondations ont des conséquences qui ne sont pour l'instant pas mesurables sur la production de documents d'archives par ces institutions. Cependant, la présence d'archivistes dans la majorité des établissements devrait enfin permettre de pouvoir anticiper ces impacts, et ce pour la première fois dans l'histoire des universités franciliennes.

\section{NOTES}

1. - Nous citerons à ce propos les contributions de: CHAPOULIE, Jean-Michel, FRIDENSON, Patrick, PROST, Antoine (dir). "Jalons pour une histoire sociale de la science et des établissements d'enseignement supérieur en France depuis 1945 ». Le Mouvement social, 2010-4 et l'article de: PICARD, Emmanuelle. "L'histoire de l'enseignement supérieur en France. Pour une approche globale ». Histoire de l'Éducation, 122, avril-juin 2009.

2. - Des précisions seront apportées sur ce point dans le cadre de la publication des actes de la journée d'étude du 8 juin 2011 intitulée Nouvelles sources pour l'histoire de l'enseignement et de la recherche: les archives universitaires.

3. - Voir à ce sujet le guide du chercheur de : CHARMASSON, Thérèse. Histoire de l'enseignement XIXe-XXe siècles, seconde édition revue et augmentée, co-éditée par l'institut national de recherche pédagogique et le comité des travaux historiques et scientifiques, Paris, 2006.

4. - Archivistes des universités, rectorats, organismes de recherche et (d'études sur les mouvements) étudiants. Ce réseau, est devenu en 2011 une section de l'AAF.

5. - Citation extraite de la circulaire du ministère de l'Éducation nationale du 27 décembre 1932 aux préfets relative au versement aux Archives départementales des archives universitaires antérieures à 1890 .

6. - Par décret du 10 juillet 1936, le versement des archives par tous les ministères, administrations, services et établissements de l'État est rendu obligatoire et toute destruction d'archives est soumise au visa de la direction des Archives de France.

7. - Circulaire $\mathrm{n}^{\circ} 70-215$ du 28 avril 1970, BOEN, nº 20 du 14 mai 1970, p. 1617-1618.

8. - MARCHAND, Marie-Louise et DUCHEIN, Michel. «Les archives de l'enseignement supérieur en France ». La Gazette des archives, nouvelle série, n 57, 2ème trimestre 1967, p. 89-111, reproduit par CHARMASSON, Thérèse (prés.). Dans Archives et sources pour l'histoire de l'enseignement. Paris : Comité des travaux scientifiques et historiques, 2005, p. 317-341.

9. - La présentation détaillée de cette enquête est parue dans BENREKASSA, H. et CHARMASSON, Thérèse. "Archives des administrations et des établissements scolaires : bilan de dix ans de versements ». Histoire de l'éducation, $n^{\circ} 18,1983$; reproduit par CHARMASSON, Thérèse (prés.). Dans Archives et sources pour l'histoire de l'enseignement. Paris : Comité des travaux scientifiques et historiques, 2005, p. 345-378.

10. - "Collecter, organiser, valoriser les archives de la recherche en sciences humaines et sociales: quelles collaborations mettre en œuvre?", journée d'étude à Aix-en-Provence 
organisée par la MSH les 18 et 19 septembre 2008, communication d'H. Gay et de S. Méchine : La place des archives des sciences humaines et sociales dans les archives des universités.

11. - PICARD, Emmanuelle. «Les archives de l'enseignement supérieur français à l'époque contemporaine : un chantier d'avenir? ». Dans MAILFERT, M.-J. et ROLLET, L. (dir.). Mémoire et culture matérielle de l'Université. Nancy : Presses universitaires de Nancy, 2008, p. 51-62.

12. - Pierre Renouvin, né en 1893, avait été nommé à la faculté des lettres de Paris dès 1933, il y était resté jusqu'en 1964 et avait occupé, de 1955 à 1958, le poste de doyen.

13. - «Les archives de l'enseignement: le rapport Renouvin» en 1969, reproduit par CHARMASSON, Thérèse (prés.). Dans Archives et sources pour l'histoire de l'enseignement. Paris : Comité des travaux scientifiques et historiques, 2005, p. 131-153.

14. - Histoire de l'éducation, $n^{\circ} 18,1983$; reproduit par CHARMASSON, Thérèse (prés.). Dans Archives et sources pour l'histoire de l'enseignement, op. cit., p. 362.

15. - Les archivistes en poste dans les universités signaleront dans leur guide des sources les quelques fonds facultaires encore conservés dans leur établissement.

16. - CHARMASSON, Th. "La mission des archives du rectorat de Paris", reproduit par CHARMASSON, Thérèse (prés.). Dans Archives et sources pour l'histoire de l'enseignement, op. cit., p. 309-316.

17. - Le classement du fonds de la Faculté de médecine conservé aux Archives nationales sous les cotes $\mathrm{AJ}^{16}$ 6226-6944 a été effectué, à la fin des années 1970, par Thérèse Charmasson.

18. - Le répertoire a été établi par D. Peycéré et est conservé sous la cote 19930551.

19. - Répertoire établi par S. Méchine et conservé sous la cote 19960475, complété et enrichi par L. Bouvier à la suite du versement des papiers versés par Jacques Sautel en 2011. Une collecte d'archives orales a été réalisée en 1995 par des étudiants de l'École normale et est conservée aux Archives nationales.

20. - Le sauvetage des archives de Jussieu a été conduit par une équipe de six personnes au départ mais plus particulièrement par Thérèse Charmasson et Stéphanie Méchine. Leur récit et la découverte d'un fonds de premier plan pour l'histoire des mathématiques ont fait l'objet de trois articles dont le dernier en date est : MÉCHINE, Stéphanie. « Archives et amiante : l'expérience de la sauvegarde des archives du campus de Jussieu dans le cadre d'un désamiantage ». Dans CHOFFEL-MAILFERT, Marie-Jeanne et ROLLET, Laurent (dir.). Mémoire et culture matérielle de l'Université : sauvegarde, valorisation et recherche, op.cit., p. 81-93.

21. - Signalons la mise à jour régulière de L'État des fonds et des versements du rectorat de Paris consultable au centre d'accueil et de recherche des Archives nationales (CARAN), 11, rue des Quatre-Fils, 75003 Paris.

22. - Article de Th. Charmasson et S. Méchine à paraître dans les actes du colloque «L'État et l'éducation, 1808-2008 » organisé par l'université Paris-IV, l'université Lille-III, et le Service d'histoire de l'éducation à Paris, 11-13 mars 2008.

23. - Une partition du fonds académique a été engagée qui a abouti au versement intégral des archives de l'enseignement scolaire, du cabinet du recteur, du secrétariat général et d'une partie de celles émanant de l'enseignement supérieur, aux Archives de Paris.

24. - Fonds conservés par la Cité des mémoires étudiantes, par la BDIC, le PAJEP, etc.

25. - Circulaire du 2 novembre 2001 relative à la gestion des archives dans les services et établissements publics de l'État, NOR : PRMX0105139C - JO du 04-11-2001, p. 17359-17360.

26. - La loi du 12 novembre 1968 sur l'orientation de l'enseignement supérieur dite loi Faure réforme l'enseignement universitaire selon trois axes: autonomie universitaire renforcée, participation étudiante aux conseils universitaires par des délégués élus, pluridisciplinarité et place accrue donnée aux aspects d'enseignement.

27. - Circulaire $\mathrm{n}^{\circ}$ 75-001du 2 janvier 1975 publiée au Bulletin officiel du ministère de 1'éducation du 16 janvier 1975, concernant les archives des examens et des bourses, élaborée en accord avec la direction des Archives de France. 
28. - Citons les universités de Paris-XII UPEC, Paris-XIII Villetaneuse, Cergy, Marne-La-Vallée, Évry et Versailles.

29. - La « palme » revient à l'université Paris-I qui est éclatée sur près de 30 sites.

30. - «Instruction DPACI/RES/2005/003 du 22 février 2005 pour le tri et la conservation des archives reçues et produites par les services et établissements concourant à l'éducation nationale ", Dans Bulletin officiel de l'Éducation nationale, $\mathrm{n}^{\circ} 24,16$ juin 2005.

31. - Loi $n^{\circ}$ 2007-1199 du 10 août 2007 relative aux libertés et responsabilités des universités parue au Journal Officiel $n^{\circ} 185$ du 11 août 2007.

32. - Présence d'asbestose sur le campus Jussieu constatée à partir des années 1970 et procès de santé publique débutés entre 2004 et 2006.

33. - Dont essentiellement les documents des directions des ressources humaines, de la scolarité, des finances, et des pôles de pilotage (Direction générale des services, Présidence).

34. - Notamment les procès-verbaux et pièces annexes des conseils d'administration, conseils scientifiques, conseils des études et de la vie universitaire, conseils des unités de formation et de recherche, etc.

35. - Rappelons à cette occasion que les rectorats ont à leur tête un recteur également chancelier des universités.

\section{RÉSUMÉS}

Le patrimoine écrit des universités suscite depuis peu l'intérêt de la communauté scientifique comme celui de ces établissements d'enseignement supérieur et de recherche tant en raison de leur intérêt administratif et juridique que de leur richesse patrimoniale. Nous nous attacherons à examiner de plus près les fonds conservés par les services d'archives définitives compétents (Archives nationales, Archives départementales), ceux collectés par le rectorat de Paris et enrichis de l'apport des archives rectorales de tutelle. Puis, dans un second temps, nous analyserons les changements institutionnels et réglementaires qui ont présidé à la création de services d'archives dans les universités et à la mise en place d'une politique de collecte des archives administratives, pédagogiques et scientifiques, notamment à travers la création du réseau professionnel Aurore et de son groupe de travail parisien.

The written heritage of french and particularly parisian universities has recently generated interest from the scientific community as well as higher education and research institutions owing to the legal, administrative historical value of these documents. We will first of all focus on the collections held by national and departmental archives institutions, and those collected by the Parisian education authority, the Rectorat of Paris, enriched by the fonds of the education supervision authority (ministry for national education, higher education and research). Then, we will try to analyze the institutional and regulatory changes that led to the creation of archives departments in universities, together with the establishment of a collecting policy for administrative, educational and scientific records, including through the creation of the AURORE national network and its professional working group in Paris. 
INDEX

Mots-clés : classement, collecte, communication, création de postes, enquête nationale, faculté, rectorat, réorganisation administrative, réseau AURORE, sources d'archives, structures archives en établissement, université

\section{AUTEURS}

\section{CHARLOTTE MADAY}

Responsable du service des archives de l'université Paris-VII (Paris-Diderot) charlotte.maday@paris7.jussieu.fr

\section{STÉPHANIE MÉCHINE}

Responsable du service des archives du rectorat de Paris stephanie.mechine@ac-paris.fr 